\title{
PAPEL ACTUAL DE LA TERAPIA COMBINADA EN LOS SÍNTOMAS DEL TRACTO URINARIO INFERIOR (STUI) MASCULINO
}

\author{
Doreen E. Chung' y Steven A. Kaplan².
}

'Fellow - Voiding Dysfunction. Incontinence. Female Urology. Weill Cornell Medical College \& Memorial SloanKettering Cancer Center

2Professor of Urology at Weill Medical College and Chief. Institute of Bladder and Prostate Health at New York Presbyterian Hospital. Cornell University. New York. USA

Resumen.- El tratamiento de los síntomas tracto urinario inferior (STUI) secundarios a hiperplasia benigna de la próstata (HBP) ha evolucionado desde el tratamiento médico quirúrgico a la monoterapia y a la terapia combinada. La terapia de primera línea médica para los hombres con STUI siguen siendo los agentes que disminuyen la resistencia de salida: $\alpha$-adrenérgicos y los inhibidores de la $5 a$-reductasa. Los agentes alfa-adrenérgicos disminuyen el tono del músculo liso de la próstata y cuello vesical. El mecanismo de acción del inhibidor de la $5 \alpha$-reductasa es la reducción en el volumen de la próstata. La terapia de primera línea para los síntomas de vejiga hiperactiva $(\mathrm{VH})$ son los agentes antimuscarínicos. Ha habido renuencia a prescribir estos fármacos a hombres con HBP, debido a la percepción de riesgo de precipitar la retención urinaria. Alfa-antagonis-

\section{CORRESPONDENCIA}

Steven A. Kaplan, MD*

Professor of Urology

Chief, Institute for Bladder and Prostate Health

Weill Cornell Medical College

Cornell University

1300 York Ave, Box 261, Suite F9 West

New York, NY 10065 (USA)

kaplans@med.cornell.edu

Aceptado para publicar: 1 de octubre 2009. tas adrenérgicos, inhibidores de la 5 a-reductasa, y los agentes antimuscarínicos, han demostrado ser seguros y eficaces cuando se administran a los hombres con HBP y STUI. La combinación de los inhibidores de la 5a-reductasa con $\alpha$-antagonistas adrenérgicos es eficaz en varones con STUI secundarios a HBP. La combinación de los antagonistas $\alpha$-adrenérgicos más antimuscarínicos y la combinación de inhibidores 5a-reductasa con antimuscarínicos son seguros y eficaces en pacientes con STUI, evidencia de la HBP y síntomas de vejiga hiperactiva. En la actualidad los inhibidores de la $5 a$ y los antagonistas $\alpha$-adrenérgicos se recomiendan en la práctica clínica. Los estudios de "Medical Therapy Of Prostatic Symptoms" (MTOPS) y "COMBinación de Avidart y Tamsulosina" (comBAT), han demostrado la superioridad de la terapia combinada sobre la monoterapia en la prevención de la progresión de la enfermedad. Se requieren más estudios para aclarar qué población específica de pacientes, se beneficiaría más de las terapias de combinación particular.

Palabras clave: Hiperplasia prostática benigna. Vejiga urinaria. Hiperactiva. alfa-antagonistas adrenérgicos. Antagonistas colinérgicos.

Summary.- Treatment of LUTS from BPH has evolved from surgical therapy to medical monotherapy to combination therapy. First-line medical therapy for men with LUTS remains agents that decrease outlet resistance: $\alpha$ adrenergic antagonists and 5a-reductase inhibitors. Alpha-adrenergic agents decrease smooth muscle tone in the prostate and bladder neck. The mechanism of action of 5 -reductase inhibitor is reduction in prostate volume. First-line therapy for $O A B$ symptoms are antimuscarinic agents. There has been reluctance to prescribe these agents to men with $\mathrm{BPH}$ due to the perceived risk of 
precipitating urinary retention. Alpha-adrenergic antagonists, $5 \alpha$-reductase inhibitors, and antimuscarinic agents have all been shown to be safe and effective when administered to men with BPH and LUTS. The combination of $5 \alpha$-reductase inhibitors with $\alpha$-adrenergic antagonists is effective in men with LUTS secondary to BPH. The combination of $\alpha$-adrenergic antagonists plus antimuscarinic agents and the combination of $5 \alpha$-reductase inhibitors with antimuscarinic agents are safe and effective in patients with LUTS, evidence of $B P H$, and $O A B$ symptoms. At present only combination therapy with $5 \alpha$ reductase inhibitors with $\alpha$-adrenergic antagonists is recommended in clinical practice guidelines. MTOPS and ComBAT have demonstrated superiority of combination therapy over monotherapy in preventing disease progression. Further studies are required to elucidate which specific patient population benefits most from particular combination therapies.

Keywords: Prostatic hyperplasia. Benign. Urinary bladder. Overactive. Adrenergic alpha-antagonists. Cholinergic antagonists.

\section{INTRODUCCIÓN}

Los síntomas del tracto urinario bajo (STUI) y la hiperplasia prostática benigna (HBP) son muy comunes en los hombres. En el estudio EPIC, una encuesta telefónica de sección transversal de 19.615 adultos en 5 países, el $62,5 \%$ de los hombres refieren tener uno o más STUI (1). El tratamiento y la comprensión de los STUI en los hombres han experimentado una importante progresión. Anteriormente, los STUI en hombres mayores se consideraban una consecuencia directa del aumento del volumen prostático y una resistencia uretral elevada. Estos síntomas se conocen como "prostatismo" y antes de la década de 1980, los hombres con STUI eran tratados principalmente con cirugía (2). En las últimas 2 décadas, la terapia médica se ha convertido en la modalidad más común de tratamiento para la HBP. La terapia puede estar dirigida a un tratamiento de los síntomas y/o a prevenir la progresión de la enfermedad.

La terapia de primera línea médica para los hombres con STUI siguen siendo los agentes que disminuyen la resistencia uretral: $\alpha$-antagonistas adrenérgicos y los inhibidores de la $5 \alpha$-reductasa $(3,4)$. Los agentes alfabloqueantes disminuyen el tono del músculo liso en la próstata y cuello de la vejiga (5). El mecanismo de acción de los $5 \alpha$-inhibidores de la reductasa es la reducción en el volumen de la próstata (5). Estudios recientes han demostrado que los inhibidores de la fosfodiesterasa tipo 5, que también inducen la relajación del músculo liso del tracto urina- rio y aunque más comúnmente utilizados para tratar la disfunción eréctil también son eficaces en el tratamiento de los STUI masculinos (6).

El tratamiento médico para los STUI en los hombres ha evolucionado, con una mejor, aunque lejos de ser absoluta, comprensión de la fisiopatología de la enfermedad. Hay nuevas evidencias de que los STUl en el hombre se produce como consecuencia de afecciones tanto de la vejiga como de la próstata. La definición del síndrome de vejiga hiperactiva (SVH) de la Sociedad Internacional de Continencia (ICS) es "la urgencia, con o sin incontinencia de urgencia, habitualmente con polaquiuria y nicturia" (7). No se comprende muy bien el vínculo entre la HBP y los síntomas de vejiga hiperactiva (8). Incluso después del tratamiento con $\alpha$-bloqueantes y con inhibidores de la $5 \alpha$-reductasa, muchos pacientes con HBP sufren de síntomas persistentes de SVH (9). El puntal del tratamiento farmacológico para la SVH es el tipo de medicamentos antimuscarínicos, inhibidores competitivos de los receptores muscarínicos. Ha habido renuencia a prescribir estos fármacos a los hombres con HBP, debido al riesgo de precipitar la retención urinaria a través de la disminución de la contractilidad de la vejiga en el marco de obstrucción del tracto urinario inferior (10). Sin embargo, diversos estudios han apoyado la eficacia y seguridad de los antimuscarínicos en el tratamiento de hombres con STUI (1 1-14).

Contraponiendo la cirugía con la monoterapia con fármacos, la siguiente progresión en el tratamiento de los STUI masculinos fue el concepto de la terapia combinada. El estudio MTOPS, un estudio aleatorizado, doble ciego y controlado con placebo de 3087 pacientes, se distribuyó a, monoterapia con alfabloqueantes, monoterapia con inhibidores $5 \alpha$ - de reductasa, o lcombinación durante 4,5 años. De demostró de la manera clara que la terapia combinada puede ser más eficaz que la monoterapia en la mejoría de los síntomas (15). Desde entonces, otros estudios han demostrado la eficacia de la terapia combinada para el tratamiento de los STUI en los hombres $(11,13,14,16)$. El objetivo de este artículo es ofrecer una revisión actual del papel de la terapia combinada en el tratamiento de los STUI masculinos.

\section{MONOTERAPIA}

Para proporcionar una mejor apreciación del papel de la terapia combinada en el tratamiento de los STUI masculinos, revisaremos en primer lugar los conceptos clave y los estudios de los fármacos individuales que se utilizan en terapia combinada. Como se mencionó anteriormente los objetivos del tratamiento 
médico son de dos tipos: el alivio de los síntomas y la prevención de la progresión de la enfermedad.

\section{Antagonistas $\alpha$-adrenérgicos (Alfabloqueantes)}

Los alfabloqueantes se consideran el tratamiento de primera línea para los STUI masculinos (3, 4). Se ha formulado la hipótesis de que la HBP causa obstrucción urinaria inferior (OUI), y síntomas a través del aumento en la estimulación $\alpha$ adrenérgica y el consiguiente aumento de tono del músculo liso uretral y la presión intrauretral. Los alfabloqueantes se utilizan para tratar los síntomas de aumento de la resistencia uretral funcional, como la HBP.

Los principales alfabloqueantes utilizados para este propósito son alfuzosina, doxazosina, tamsulosina y terazosina. En la próstata y la uretra masculina, el subtipo de receptor $\alpha 1 \mathrm{~A}$ es el más prevalente. Todos estos agentes son selectivos para el subtipo de receptor $\alpha 1$ presente en el tejido prostático. La tamsulosina es el único $\alpha 1 \mathrm{~A}$ selectivo $\alpha$-bloqueante adrenérgico. Sin embargo, ningún agente único ha demostrado ser significativamente más eficaz que los otros.

El Comité de Directrices de Práctica Clínica de la AUA realizó un meta-análisis y concluyeron que los alfabloqueantes eran beneficiosos en el tratamiento de la OUl y la hiperactividad del detrusor por HBP (3). En general, los $\alpha$-bloqueantes mejoran, en 4-6 puntos, el índice de síntomas de la AUA (AUASI). Numerosos estudios han confirmado la eficacia y tolerabilidad de los antagonistas $\alpha$-adrenérgicos (17-19). Se ha informado de efectos secundarios en aproximadamente 5 a $9 \%$ de las poblaciones de pacientes que toman adrenérgicos $\alpha$-antagonistas (20). Estos incluyen mareos, hipotensión postural, astenia, rinitis, y disfunción sexual, incluida la eyaculación retrógrada.

Elhilali et al., llevaron a cabo un estudio multicéntrico aleatorizado doble ciego controlado con placebo de 164 pacientes con HBP para investigar la seguridad y la eficacia de terazosina. A las 24 semanas la terazosina aumentó significativamente el caudal máximo $(p<0,001)$ y no alteró el residuo post-miccional (RPM). También hubo una mejora significativa en el AUASI ( $p=0,014)$ (17). Sólo 11 pacientes abandonaron el estudio debido a episodios adversos: 7 del grupo de terazosina y 4 del grupo placebo.

Un análisis combinado de los tres, doble ciego, controlado con placebo con una población combinada de 337 sujetos se llevó a cabo para evaluar la seguridad y la eficacia de la doxazosina en pa- cientes con HBP. La doxazosina produjo una mejoría significativamente mayor que el placebo en la tasa máxima de flujo urinario $(P=0,0017)$, gravedad de los síntomas ( $p<0,0001)$, y molestias causadas por los síntomas ( $p<0,0001$ ) (19). En general, el medicamento fue bien tolerado. El $10 \%$ de los pacientes en el grupo de tratamiento suspendió la medicación frente al $4 \%$ en el grupo placebo $(p<0,05)$.

La seguridad y eficacia de alfuzosina una vez al día para el tratamiento de los STUI debido a la HBP se evaluó en un análisis combinado de tres estudios paralelos doble ciego, controlados con placebo. Los pacientes fueron aleatorizados a tomar alfuzosina $10 \mathrm{mg}$ una vez al día o placebo durante 12 semanas. La alfuzosina mejoró significativamente el IPSS total ( $p<0,005)$, la sub-valoración del IPSS irritativo ( $p<0,001)$, sub-valoración del IPSS obstructivo crónico ( $p<0,001)$, y el $Q \max (p<0,001)$ en comparación con el placebo. Las tasas de abandono fueron bajas y comparables a las de placebo (21).

Se realizó un ensayo aleatorio doble-ciego controlado por placebo de tamsulosina 0,4 mg, tamsulosina $0,8 \mathrm{mg}$ y placebo para determinar la seguridad y la eficacia de la tamsulosina en el tratamiento de los STUI en hombres con HBP (22). Se incluyeron 756 sujetos durante 13 semanas. Se observaron mejorías significativas en AUASI y Qmáx en ambos grupos de tratamiento. En general, se informaron de más episodios adversos en el grupo de tamsulosina 0,8 $\mathrm{mg}$, en comparación con el de placebo. Sin embargo, la incidencia de episodios adversos en el grupo de 0,4 mg fue comparable a la de placebo.

\section{Inhibidores de la $5 \alpha$-reductasa (5ARI)}

La enzima $5 \alpha$-reductasa convierte la testosterona en dihidrotestosterona (DHT). La castración y los agentes farmacológicos que suprimen la testosterona y/o la secreción de DHT se ha demostrado que reduce el tamaño de la próstata en hombres con HBP (23). Se ha formulado la hipótesis de que la reducción de volumen de la próstata disminuye el componente estático de las OUI causada por HBP. Los inhibidores $5 \alpha$-reductasa, finasterida y dutasterida son seguros y eficaces en el tratamiento de la HBP (26-30). La dutasterida inhibe las isoenzimas tanto la de tipo 1 como la de tipo 2, mientras que la finasterida inhibe sólo la isoenzima de tipo 2. En los estudios se ha encontrado que, en general, la $5 \alpha$-reductasa es más eficaz en pacientes con próstatas de mayor tamaño que en aquellos con próstatas más pequeñas $(24,25)$.

De los dos inhibidores de la $5 \alpha$-reductasa, la finasterida ha sido el más ampliamente estudiado. 
Los primeros ensayos aleatorizado, doble-ciego controlado con placebo de finasterida versus placebo, en 895 y 750 pacientes, fueron de sólo 1 año de duración $(26,27)$. Sin embargo se demostró que la finasterida mejoró significativamente las puntuaciones de los síntomas ( $p<0,001$ y $p<0,015$ ) y $Q \max$ ( $p<0,001)$ en comparación con placebo después de 12 meses de tratamiento. Un meta-análisis de estos primeros estudios llegó a la conclusión de que estas mejorías fueron bastante menores en los pacientes con próstatas más pequeñas (25).

El estudio de eficacia y seguridad a nivel mundial Proscar (PROWESS) fue un ensayo de 2 años multicéntrico aleatorizado doble ciego controlado con placebo para demostrar la eficacia y la seguridad de finasterida en el tratamiento de hombres con STUI por HBP (28). Los resultados de estos estudios de corta duración fueron confirmados por un ensayo de 4 años aleatorizado, doble ciego controlado por placebo, el Estudio de Eficacia y Eeguridad de Larga Duración Proscar (PLESS) (29). El PLESS demostró que la finasterida redujo significativamente el riesgo de retención urinaria $(p<0,001)$, así como la necesidad de cirugía $(p<0,001)$ en comparación con el placebo en pacientes con próstatas mayores de $55 \mathrm{~g}$. El finasteride fue bien tolerado y la tasa de abandono fue similar a la de placebo.

Roehrborn et al., probaron la seguridad y eficacia de la dutasterida en un ensayo aleatorizado doble ciego controlado por placebo (30). 4325 pacientes con STUI y HBP, con síntomas moderadosgraves, con un Qmáx $15 \mathrm{~mL} / \mathrm{s}$ o menos, $30 \mathrm{cc}$ de volumen de la próstata 0 más, fueron aleatorizados con dutasterida $0,5 \mathrm{mg}$ o placebo durante 24 meses. La puntuación de los síntomas mejoró significativamente a partir de 6 meses en adelante $(p<0,001)$ con una mejoría media de 4,5 puntos en 24 meses. El Qmáx mejoró de forma importante a partir del 1 mes en adelante $(p<0,01)$. La tasa de flujo máximo se incrementó significativamente a partir de 1 mes $(P$ $<0,01)$, con un aumento de $2,2 \mathrm{~mL} / \mathrm{s}$ informado a los 24 meses $(P<0,001)$. La reducción del riesgo de retención urinaria aguda fue del $57 \%$ y la reducción del riesgo de intervención quirúrgica fue de $48 \%$ en comparación con el placebo. La impotencia, disminución de la libido, trastornos de la eyaculación, y la ginecomastia se produjeron con más frecuencia en los pacientes tratados con dutasterida. En general, el fármaco fue bien tolerado.

\section{Antimuscarínicos}

Estudios poblacionales como el estudio EPIC han demostrado que en los hombres los síntomas irri- tativos de vejiga hiperactiva $(\mathrm{VH})$ son más frecuentes que los síntomas de vaciado o post-miccionales y que se asocian con un alto grado de molestia. Curiosamente, en los estudios urodinámicos de los hombres con STUI, sólo 48-68\% tienen OUI $(31,32)$.

La terapia de primera línea para los síntomas de $\mathrm{VH}$ son los agentes antimuscarínicos. Ha habido renuencia a prescribir estos fármacos a hombres con $\mathrm{HBP}$, debido al riesgo de precipitar la retención urinaria a través de la disminución de la contractilidad de la vejiga en el marco de obstrucción del tracto urinario inferior (10). Sin embargo, diversos estudios han apoyado la eficacia y seguridad de los antimuscarínicos en el tratamiento de hombres con STUI (1 1 $14,16)$. Las actuales guías de práctica no recomiendan el uso de anticolinérgicos en hombres con STUI sugerentes de $\operatorname{HBP}(3,4)$.

De los 1529 pacientes con síntomas de vejiga hiperactiva inscritos en un estudio aleatorizado de seguridad y eficacia de tolterodina de liberación prolongada (ER), tolterodina de liberación inmediata $(I R)$, y placebo, el $19 \%$ eran hombres (33). Las tolterodina $E R$ y $I R$ redujeron significativamente el número medio de episodios de incontinencia por semana en comparación con el placebo y la formulación ER fue un $18 \%$ más eficaz que la formulación de IR ( $p$ $<0,05)$. Los índices de retirada fueron similares al placebo en ambos grupos de tolterodina.

Se realizó un estudio aleatorizado doble ciego controlado con placebo de succinato de solifenacina para evaluar la seguridad y eficacia (34). 911 hombres y mujeres (155, 17\% de hombres) con síntomas de vejiga hiperactiva fueron aleatorizados a placebo, $5 \mathrm{mg}$ de solifenacina, o $10 \mathrm{mg}$ de solifenacina. Después de 12 semanas, las 24 horas de frecuencia disminuyó significativamente con $5 \mathrm{mg}$ de solifenacina $(-2,37, p=0,0018)$ y $10 \mathrm{mg}$ de solifenacina $(-2,81, p=0,0001)$. En los grupos de solifenacina también hubo una disminución significativa en episodios de incontinencia $(5 \mathrm{p}=0,002 \mathrm{mg}, 10 \mathrm{mg} \mathrm{p}$ $=0,016)$, episodios de incontinencia de urgencia $(5$ $\mathrm{mg}, \mathrm{p}=0,014$ y $10 \mathrm{mg}, \mathrm{p}=0,042)$, y episodios de urgencia $15 \mathrm{mg}-2,84 \mathrm{p}=0,003,10 \mathrm{mg}-2,90 \mathrm{p}=$ $0,002)$. La terapia fue bien tolerada. La incidencia de boca seca leve fue de $2,3 \%$ en el placebo, el $7,7 \%$ en el grupo de $5 \mathrm{mg}$ de solifenacina, y $23 \%$ en el grupo de $10 \mathrm{mg}$ de solifenacina.

Abrams et al., estudiaron el efecto de la tolterodina IR en hombres con evidencia tanto de OUI como de $\mathrm{VH}(13)$. Los criterios de inclusión fueron la frecuencia urinaria (8 o más micciones en 24 horas), urgencia (1 o más episodios en24 horas) y el índice de OUI 20 o mayor. 222 hombres fueron asignados 
al azar con $I R$ tolterodina $2 \mathrm{mg}$ dos veces al día o placebo durante 12 semanas. Las diferencias promedio del tratamiento en Qmáx $1-0,7 \mathrm{ml}$ por segundo, del $95 \%$ : $-1,6$ a 0,4$)$ y $p$ det $Q \max (-7 \mathrm{~cm}$ de $\mathrm{H} 2 \mathrm{O}$, del 95\%: -3 a 11 ) fueron comparables. La tolterodina redujo significativamente el índice de OUI (-9 vs $0, p<0,02)$ e incrementó la capacidad cistométrica máxima $(67 \mathrm{ml}, \mathrm{IC} 95 \% 35-103, \mathrm{p}<0,003)$ en comparación con el placebo. El cambio en el RPM fue significativamente mayor entre los pacientes tratados con tolterodina $(25 \mathrm{ml})$ que con placebo $(0 \mathrm{ml}$, $p<0,004)$. No se apreciaron diferencias significativas en la incidencia de episodios adversos. Un solo paciente informó de retención urinaria en el grupo placebo. Estos resultados sugieren que los antimuscarínicos pueden ser administrados de forma segura en hombres con OUl y $\mathrm{VH}$.

\section{TERAPIA COMBINADA}

\section{Antagonistas $\alpha$-adrenérgicos (Alfabloqueantes) con inhibidores de la $5 \alpha$-reductasa}

Debido a las diferentes clases de tratamiento farmacológico utilizado para el tratamiento de los STUI masculinos funcionan por medio de diferentes mecanismos, parece lógico que los pacientes deben recibir más beneficios cuando son tratados con más de una clase de fármacos. Este concepto ha sido estudiado en la década de 1990, pero los primeros ensayos de la terapia combinada no han demostrado un beneficio significativo sobre la monoterapia $(34,35)$. En consecuencia, la terapia combinada no se practicó de forma amplia hasta que se publicaron los resultados del estudio MTOPS. Hoy en día, la AUA y la EAU recomiendan la terapia combinada con alfabloquentes y $5 \mathrm{ARI}$ para pacientes con síntomas moderadosgraves y aumento del tamaño prostático $(3,4)$.

El primer estudio multicéntrico aleatorizado doble ciego controlado con placebo, que evaluó la terapia combinada de los antagonistas $\alpha$-adrenérgicos y los 5ARI fue el Estudio Cooperativo de Veteranos (34). 1229 pacientes con STUI secundarios a HBP fueron aleatorizados y tratados con finasterida $5 \mathrm{mg}$, $10 \mathrm{mg}$ de terazosina, o terapia combinada durante 12 meses. No se encontraron diferencias significativas entre los grupos de placebo y la finasterida para AUASI o $Q$ máx. Sin embargo, hubo diferencias significativas entre el AUASI visto en la terazosina y los grupos de terapia combinada en comparación con el placebo $(p<0,001)$ y la finasterida $(p<0,001)$. También se observaron cambios importantes en Qmáx entre ambos terazosina y terapia combinada en comparación con placebo ( $p<0,001)$ y la finasterida ( $p$ $<0,001)$. En general, en esta población de pacientes la terapia combinada no fue más eficaz que la terazosina sola. Y, la finasterida no fue más beneficiosa que el placebo en la mejora de AUASI y Qmáx.

El estudio prospectivo europeo de doxazosina y terapia combinada (PREDICT) fue similar en diseño al Estudio de Veteranos y los resultados comparables (35). Fue un ensayo aleatorizado doble ciego controlado con placebo de 1095 pacientes a los que se asignó al azar doxazosina, finasterida, o la terapia combinada durante 1 año. Los objetivos principales fueron el cambio en el IPSS y Qmáx. Se observaron mejorías significativas en el IPSS tanto en los grupos de monoterapia de doxazosina y combinación, en comparación con los grupos de finasterida $(p<0,001)$ y placebo $(p<0,001)$. Al igual que el Estudio de Veteranos, no hubo diferencias significativas entre la finasterida y el placebo en términos de cambios en el IPSS y Qmáx. La adición de finasteride no añadió ningún beneficio adicional al de la doxazosina sola en el IPSS o Qmáx. Ambos estudios, el Estudio Cooperativo de los Veteranos y el estudio de PREDICT están limitados por la corta duración de sólo un año. Además, su principal medida de efectividad fue el alivio de los síntomas. Muchos beneficios de los inhibidores de la $5 \alpha$-reductasa se encuentran en la categoría de prevención de la enfermedad en lugar de mejorar los síntomas.

El estudio MTOPS fue de mayor duración que los estudios anteriores de la terapia combinada y sus objetivos se centraron en la progresión de la enfermedad, en lugar del alivio de los síntomas (15). El principal objetivo del estudio fue determinar si el tratamiento con doxazosina, finasterida, o una combinación puede prevenir la progresión de la enfermedad (HBP) definida por un aumento de al menos 4 puntos en el AUASI, retención aguda de orina (RAO), incontinencia, insuficiencia renal, o las infecciones urinarias recurrentes (IUR). Se asignó al azar a 3047 hombres finasteride, doxazosina, terapia combinada, o placebo, con una duración media de 4,5 años. El riesgo de progresión clínica fue significativamente reducido por la doxazosina (39 por ciento de reducción del riesgo, $P<0,001$ ) y el finasteride (34 por ciento de reducción del riesgo, $P=0,002)$, en comparación con el placebo. La reducción del riesgo en el grupo de terapia combinada (66 por ciento en comparación con el placebo, $P<0,001$ ) fue significativamente mayor que la asociada con doxazosina $(p<0,001) \circ$ con finasterida $(\mathrm{P}<0,001)$ sola. Los grupos de combinación y de finasterida mostraron una significativa disminución en el riesgo de retención urinaria aguda y la necesidad de tratamiento invasivo ( $p<0,001)$, pero esto no se observó en el grupo de doxazosina. Los efectos adversos fueron significativamente más frecuentes en el grupo de combinación. Cuando los 
pacientes fueron estratificados de acuerdo al tamaño de la próstata, se constató que en los hombres con próstatas pequeñas (menos de $25 \mathrm{ml}$ a nivel basal) la terapia combinada no fue más beneficiosa que la doxazosina sola reduciendo el riesgo de progresión clínica de la HBP, la necesidad de tratamiento invasivo, la mejora de AUASI y Qmáx (36). Sin embargo, en los hombres con próstatas de tamaño moderado (25 - $<40 \mathrm{~mL}$ ) o aumento del tamaño prostático (tamaño de $40 \mathrm{ml}$ o más) el tratamiento combinado tuvo un beneficio significativo sobre ambas monoterapias doxazosina y finasterida.

El primer estudio aleatorizado, doble-ciego controlado con placebo de la terapia combinada con un antagonista $\alpha$-adrenérgicos y del inhibidor de la $5 \alpha$-reductada (dutasterida) fue el "Symptoms Management after Reducing Therapy" (SMART) (37). Este estudio investigó si a los hombres tratados con una combinación de dutasterida y tamsulosina, seguida por la retirada de la tamsulosina puede afectar negativamente los síntomas en hombres con STUI. 327 hombres fueron asignados al azar a la terapia combinada durante 36 semanas o la terapia combinada durante 24 semanas seguidas de 12 semanas de monoterapia con dutasterida y placebo. El $77 \%$ de los pacientes de combinación con cambio al grupo de monoterapia de dutasterida sentía lo mismo o mejor en la semana 30 en comparación con la semana 24 . La principal limitación de este estudio fue la corta duración del tratamiento.

Un estudio, a largo plazo, aleatorizado, doble ciego y controlado con placebo, el "COMBinación de Avidart y Tamsulosina" (comBAT), está investigando los efectos de la dutasterida, tamsulosina, y la terapia combinada en varones con STUI secundarios a HBP (38). 4844 hombres con STUI moderada-grave (IPSS 12 o mayor) y tamaño prostático $>30 \mathrm{cc}$, fueron aleatorizados durante 4 años a dutasterida, tamsulosina, o terapia combinada. El objetivo principal del estudio a los 2 años fue el cambio en el IPSS. A los dos años, con la terapia combinada el IPSS disminuyó significativamente en comparación con el grupo de finasterida a los tres meses, y el grupo de tamsulosina a los 9 meses $(p<0,001)$. También hubo un aumento significativo en el grupo de terapia combinada del Qmáx en comparación con los grupos de monoterapia $(p \leq 0,006)$. La principal crítica de este estudio es la falta de grupo de placebo.

\section{Antagonistas $\alpha$-adrenérgicos con antimuscarínicos}

Incluso después del tratamiento con antagonistas de la $\alpha$-adrenérgicos e inhibidores de la $5 \alpha$ reductasa, muchos pacientes con HBP sufren de sínto- mas persistentes de $\mathrm{VH}$. Hay pruebas de que después del fracaso de los antagonistas $\alpha$-adrenérgicos, la adición de un agente antimuscarínico es beneficiosa y segura $(12,14,39)$. También hay pruebas de que en los hombres con STUI, síntomas de vejiga hiperactiva, y evidencia de HBP, la terapia combinada de un antagonista $\alpha$-adrenérgico con un agente antimuscarínico es eficaz y bien tolerada. Es importante señalar que la mayoría de los ensayos se han seleccionado específicamente para pacientes que no tienen evidencia de OUI importante.

Los tres primeros ensayos descritos en esta sección incluyeron pacientes con $\mathrm{OUI}$ urodinámicamente probada. Athanasopoulos et al., realizaron un estudio aleatorizado de 50 pacientes con OUI urodinámicamente probada asigndos a recibir tamsulosina solo durante 3 meses o tamsulosina solo durante 1 semana seguida de la terapia de combinación con tamsulosina y tolterodina (39). Los pacientes fueron evaluados con urodinámica y cuestionario de calidad de vida (QoL) después de 3 meses. Hubo una mejoría significativa en las puntuaciones de la calidad de vida antes y después del tratamiento sólo en el grupo de combinación, así como una disminución significativa de la presión máxima del detrusor durante la micción $(-8,24 \mathrm{~cm} \mathrm{H} 20, p=0,0082)$. Ambos grupos reflejaron una gran diferencia en el Qmáx y el volumen a la primera contracción.

En otro estudio, incluyendo a hombres con vejiga hiperactiva y OUl urodinámicamente demostrado, Lee et al., evaluaron la eficacia del tratamiento combinado con doxazosina y tolterodina IR (14). Todos los pacientes fueron tratados con doxazosina $4 \mathrm{mg}$ al día durante 3 meses. En los pacientes sin mejoría, se les añadió tolterodina IR $2 \mathrm{mg}$ dos veces al día. Estos pacientes, sin ninguna mejoría, 6 de 16 con OUl y 32 de $44(73 \%)$ con OUI + VH mejoraron tras la adición de tolterodina. Desarrollaron retención aguda de orina 2 de 60 hombres $(3,3 \%)$ tratados con la terapia combinada.

Se evalúo la seguridad y la eficacia de la combinación del tratamiento con clorhidrato de propiverina y doxazosina en un estudio prospectivo aleatorizado multicéntrico controlado, incluyendo 211 hombres con vejiga hiperactiva y $\mathrm{OUI}$ urodinámicamente demostrada (40). Los pacientes fueron aleatorizados con doxazosina o doxazosina más propiverina durante 8 semanas. Hubo mejoría importante en el grupo de combinación en comparación con el grupo de monoterapia en la frecuencia $(p=0,001)$, síntomas de almacenamiento $(p=0,029)$, satisfacción del paciente $(p=0,002)$. El RPM aumentó significativamente en el grupo de combinación $(20.8 \mathrm{~mL}, \mathrm{p}=0,002)$ pero no se informó de pacientes con retención urinaria. 
Kaplan et al., llevaron a cabo un estudio prospectivo abierto para determinar la eficacia y seguridad de la tolterodina ER en hombres con HBP y STUI en los que la terapia previa con alfa-bloqueantes había fracasado (12). Cuarenta y tres pacientes consecutivos en los que la terapia con alfa-bloqueante había fracasado debido a eventos adversos o la falta de eficacia recibieron $4 \mathrm{mg}$ de tolterodina ER durante 6 meses. La frecuencia media de micción durante 24 horas disminuyó desde 9,8 hasta 6,3 veces y nicturia desde 4, 1 hasta 2,9 episodios por noche. También se observaron disminuciones significativas en el IPSS y RPM. Cuatro hombres (9\%) suspendieron el tratamiento debido a sequedad de boca y ninguno de los pacientes presentó retención urinaria.

En 2006, Kaplan et al., informaron sobre un gran estudio aleatorio, con placebo, doble ciego y controlado con placebo de tolterodina ER en hombres de 40 años o mayores con STUl y VH (41). Este estudio se conoce como el estudio TIMES. Los criterios de inclusión fueron: edad 40 años o más, IPSS 12 o más alto, IPSS calidad de vida de vida (QOL) puntuación de 3 o superior, autopercepción del estado de la vejiga o por lo menos moderada molestia, diario de la vejiga documentando la frecuencia miccional ( $\geq 8$ micciones en 24 horas) y urgencia ( $\geq 3$ episodios en 24 horas) con o sin incontinencia urinaria de urgencia. En otras palabras, los pacientes cumplieron con los criterios de entrada para los estudios de VH y HBP. Es de destacar que se excluyeron los pacientes con evidencia de obstrucción urinaria inferior (OUI). Los criterios de exclusión incluyeron Qmáx <5 ml/segundo, RPM> de $200 \mathrm{ml}$, e historia de retención urinaria aguda. 879 pacientes fueron aleatorizados a placebo, tolterodina ER $4 \mathrm{mg}, 0,4 \mathrm{mg}$ de tamsulosina, o ambos tolterodina ER y tamsulosina durante 12 semanas. Los pacientes del grupo de terapia combinada experimentaron una reducción significativa en incontinencia urinaria de urgencia $(p=0,005)$, episodios de urgencia sin incontinencia $(p=0,03)$, micciones en 24 horas $(p<0,001)$, micciones en la noche ( $p=$ $0,02)$, IPSS total $(p=0,003)$, y puntuación del IPSS de la calidad de vida $(p=0,003)$ en comparación con el placebo. En la semana 12, hubo mejorías importantes en el IPSS observado en la tamsulosina (p $=0,003)$ y los grupos de combinación $(p=0,003)$ en comparación con el de referencia, pero no se observó lo mismo en los grupos de placebo o tolterodina ER. La incidencia de retención urinaria aguda que requirió cateterismo fue baja en todos los grupos $10,4 \%$ tamsulosina + tolterodina $E R, 0.5 \%$ tolterodina ER\%, 0\% placebo, 0\% tamsulosina). Se observó un aumento de 5 a $6 \mathrm{ml}$ de la RPM tanto en el grupo de tolterodina ER y como en el de tolterodina ER + tamsulosina que no fue clínicamente significativo. En ninguno de los dos grupos se observaron diferencias estadísticamente importantes en la RPM.

Un sub-análisis del estudio TIMES analizó los efectos de la tolterodina ER, tamsulosina, placebo y tolterodina ER además de tamsulosina en el IPSS. Durante 12 semanas, los pacientes que recibieron tolterodina ER además de tamsulosina tenían mejoras significativas en la subescala de síntomas de llenado en el IPSS y todos los elementos en el IPSS en comparación con placebo (42). En los grupos de monoterapia, los resultados de los cambios en la subescala de llenado del IPSS e individuales no fue significativamente diferente a la de placebo. En contraste, los grupos de tolterodina ER y tolterodina ER más tamsulosina no mostraron una diferencia significativa en la subescala del IPSS de micción en comparación con placebo.

Chapple et al., evaluaron la eficacia de la tolterodina ER en 652 hombres con STUI, con una dosis estable de alfa-bloqueante durante al menos un mes, aleatorizados con tolterodina ER $4 \mathrm{mg}$ al día - placebo durante 12 semanas (43). Se encontró que los hombres con síntomas importantes de vejiga hiperactiva que ya estaban en tratamiento con alfabloqueante mostraron mejorías importantes en las variables del diario, resultados del IPSS de llenado, y síntomas al recibir tolterodina ER adicional.

Rovner et al., estudiaron la eficacia de la tolterodina ER y/o tamsulosina en los episodios de urgencia relacionados con la micción, gravedad de la urgencia, y los informes de resultados de los pacientes en hombres que cumplieron los criterios de inclusion por aumento del volumen prostático y ensayos de $\mathrm{VH}$ (44). También descubrieron que la tolterodina ER y la tamsulosina mejoraron significativamente las variables de urgencia y los resultados informados por los pacientes.

MacDiarmid et al., llevaron a cabo un ensayo aleatorizado doble ciego controlado con placebo de pacientes que tomaban tamsulosina aleatorizados a placebo o a oxibutinina de liberación prolongada (16) e informaron que la terapia combinada es segura y eficaz. Al igual que la mayoría de los estudios de antimuscarínicos en hombres con HBP, el estudio excluyó a los pacientes con OUl grave. En el grupo de tamsulosina oxibutinina ER, se produjeron mejorías significativas en la puntuación total del IPSS ( $p=$ 0,038 semanas, $p=0,00612$ semana), IPSS-dominio de llenado $(p<0,01)$, y puntuaciones de calidad de vida $(p<0,01)$ después de 8 y 12 semanas. La incidencia de la RPM mayor de $300 \mathrm{ml}$ fue baja y similar en los grupos de monoterapia con tamsulosina y de combinación. 
Se han llevado a cabo varios estudios para definir la población de hombres con STUI que más se beneficiarán de añadir tolterodina ER. Roehrborn et al., realizaron un análisis retrospectivo de hombres asignados al azar a placebo, tolterodina ER, tamsulosina, o tolterodina ER más tamsulosina que fueron estratificados de acuerdo con el PSA basal ( $\geq$ $1,3 \mathrm{ng} / \mathrm{ml}$ frente a $<1,3 \mathrm{ng} / \mathrm{ml}$ ) (45). El nivel de PSA se correlacionó significativamente con el tamaño de la próstata. En los hombres con un nivel de PSA $<1,3 \mathrm{ng} / \mathrm{mL}$, únicamente tolterodina ER, así como tolterodina ER más tamsulosina mejoraron significativamente la frecuencia de 24 horas, durante el día, la de imperiosidad miccional, y las puntuaciones de IPSS de llenado en comparación con el placebo. En pacientes con PSA $\geq 1,3 \mathrm{ng} / \mathrm{mL}$ tomando sólo tolterodina ER más tamsulosina experimentado mejorías significativas en la frecuencia de 24-horas, durante el día, imperiosidad miccional, IPSS total, y el IPSS de llenado en comparación con el placebo. En el grupo con próstatas más grandes, tratado sólo con tolterodina $E R$, no se produjeron mejorías significativas en el diario miccional o en las variables de puntuación de síntomas.

Otro análisis retrospectivo examinó el efecto del tamaño de la próstata en hombres que cumplían criterios de HBP y los estudios de $\mathrm{VH}$, aleatorizados a placebo, tolterodina $E R$, tamsulosina, o tolterodina ER más tamsulosina (46). Al igual que en el estudio anterior, los hombres con próstatas más pequeñas y de moderada a grave STUI, incluyendo síntomas de vejiga hiperactiva, se benefició de la monoterapia con tolterodina ER. El tratamiento con tolterodina ER más tamsulosina fue eficaz independientemente del tamaño de la próstata. La incidencia de retención urinaria aguda fue baja en todos los grupos $(\leq 2 \%)$.

\section{Inhibidores $5 \alpha$-reductasa y antimuscarínicos}

Hay pruebas de que la terapia combinada con inhibidores de la $5 \alpha$-reductasa y agentes antimuscarínicos es segura y eficaz en hombres con HBP y STUI (47). Chung et al., evaluaron la seguridad y la eficacia de tolterodina ER $4 \mathrm{mg}$ con dutasterida 0,5 $\mathrm{mg}$ en hombres con síntomas persistentes de vejiga hiperactiva y STUI tratados sin éxito con dutasterida solo. 51 hombres tratados con dutasterida durante un mínimo de 6 meses con síntomas persistentes de $\mathrm{VH}$ participaron en un estudio abierto de 12 semanas y se les dio tolterodina ER. Los criterios de inclusión fueron IPSS $\geq 12$, IPSS tema calidad de vida $\geq 3$, molestias importantes, frecuencia ( $\geq 8$ micciones $/ 24 \mathrm{~h}$ ) y urgencia ( $\geq 3$ episodios $/ 24 \mathrm{~h}$ ). El volumen prostático inicial fue $54,3 \mathrm{ml}$. La tolterodina ER redujo significativamente la frecuencia y urgencia: frecuencia mic- cional 24 horas $(-3,2, p<0,02)$, episodios de $\mathrm{VH}$ $(19,2 \%, p<0,03)$, episodios graves de $\mathrm{VH}(71,4 \%$, $p<0,05)$, micción nocturna $(-0,9, p<0,003)$. El IPSS disminuyó con la monoterapia con dutasterida (19.3 a 14.3) y disminuyó añadiéndole tolterodina a $7,1(p<0,001)$.

Los síntomas de almacenamiento disminuyeron de 9,8 a $3,5(p<0,001)$. La boca seca se produjo en $4(7,5 \%)$ sujetos, estreñimiento en $1(2 \%)$ y disminución de la función sexual en $2(3,9 \%)$. El RPM aumentó en 4,2 ml, Qmáx disminuyó en 0,2 $\mathrm{ml} / \mathrm{seg}$ y ningún paciente sufrió retención. La combinación Tolterodina y Dutasteride fue eficaz, segura y bien tolerada en hombres con próstatas grandes $1 \geq 30$ $\mathrm{mL}$ ) con síntomas persistentes de vejiga hiperactiva y STUI secundarios a HBP.

\section{CONCLUSIONES}

El tratamiento de los STUI secundarios a HBP ha evolucionado desde el tratamiento quirúrgico a la monoterapia y a la terapia combinada. La combinación de los inhibidores de la $5 \alpha$-reductasa con $\alpha$-antagonistas adrenérgicos es eficaz en varones con STUI secundarios a HBP. La combinación de los antagonistas $\alpha$-adrenérgicos más antimuscarínicos y la combinación de inhibidores $5 \alpha$-reductasa con antimuscarínicos son seguros y eficaces en pacientes con STUI, evidencia de HBP y síntomas de vejiga hiperactiva. En la actualidad sólo se recomienda en las guías de práctica clínica, la terapia combinada con inhibidores de la $5 \alpha$-reductasa con antagonistas $\alpha$-adrenérgicos. Los grandes estudios a largo plazo MTOPS y ComBAT han demostrado la superioridad de la terapia combinada sobre la monoterapia en la prevención de la progresión de la enfermedad. Se requieren más estudios para aclarar que población específica de pacientes se beneficia más de las terapias combinadas en particular.

\section{BIBLIOGRAFIA y LECTURAS RECOMENDADAS (*lectura de interés $y^{* *}$ lectura fundamental)}

1. Irwin D, Milsom I, Hunskaar S, et al. Populationbased survey of urinary incontinence, overactive bladder, and other lower urinary tract symptoms in five countries: results of the EPIC study. Eur Urol. 2006; (50):1306-14.

2. Kirby R, Lepor H. Evaluation and non-surgical management of benign prostatic hypeplasia. In: Wein A, Kavoussi L, Novick A, Partin A, Peters C, editors. Campbell-Walsh Urology, 9th ed. Philadelphia: Saunders Elsevier; 2007. 
3. Guideline on the Management of Benign Prostatic Hyperplasia (HBP). Journal [serial on the Internet]. 2003 Date.

4. de La Rosette J, Alivizatos G, Madersbacher M, Rioja Sanz C, Nordling J. Guidelines on benign Prostatic Hyperplasia. Journal [serial on the Internet]. 2004 Date.

5. Chapple C. Pharmacological therapy of benign prostatic hyperplasia/lower urinary tract symptoms: an overview for the practising clinician. BJU International. 2004;94:738-44.

6. McVary K, Roehrborn C, Kaminetsky J, al e. Tadalafil relieves lower urinary tract symptoms secondary to benign prostatic hyperplasia. J Urol. 2007;177:1404-7.

7. Abrams P, Cardozo L, Fall M, al. e. The standardization of terminology in lower urinary tract function: report from the standardization sub-committee of the International Continence Society. Urology. 2003;61:37-49.

8. Blake-James B, Rashidian A, Ikeda Y, Emberton $M$. The role of anticholinergics in men with lower urinary tract symptoms suggestive of benign prostatic hyperplasia: a systematic review and metaanalysis. BJU International. 2007;99(1):85-96.

9. Chapple C, Roehrborn C. A shifted paradigm for the further understanding, evaluation, and treatment of lower urinary tract symptoms in men: focus on the bladder. Eur Urol. 2006;49:651-9.

10. McGuire E. Neuromuscular dysfunction of the lower urinary tract In: Walsh P, Gittes R, Perlmutter A, Stamey T, editors. Campbell's Urology. Philadelphia: W. B. Saunders; 1986.

**11. Kaplan S, Roehrborn C, Rovner E, Carlsson M, Bavendam T, Guan Z. Tolterodine and tamsulosin for treatment of men with lower urinary tract symptoms and overactive bladder: a randomized controlled trial. JAMA. 2006;296(19):2319-28.

12. Kaplan S, Walmsley K, Te A. Tolterodine extended release attenuates lower urinary tract symptoms in men with benign prostatic hyperplasia. J Urol. 2008;179(Suppl 5):S82-5.

**13. Abrams P, Kaplan S, De Koning Gans H, Millard R. Safety and tolerability of tolterodine for the treatment of overactive bladder in men with bladder outlet obstruction. J Urol. 2006;175(3):999-1004

14. Lee J, Kim H, Lee S, Koh J, Suh H, Chancellor M. Comparison of doxazosin with or without tolterodine in men with symptomatic bladder outlet obstruction and an overactive bladder. BJU International. 2004;94(6):817-20.

**15. McConnell J, Roehrborn C, Bautista O, Andriole JL, et al. Group tMToPSMR. The long-term effect of doxazosin, finasteride, and combination therapy on the clinical progression of benign prostatic hyperplasia. NEJM. 2003;349:2387-98.

16. MacDiarmid S, Peters K, Chen A, Armstrong R,
Orman C, Aquilina J, et al. Efficacy and safety of extended-release oxybutynin in combination with tamsulosin for treatment of lower urinary tract symptoms in men: randomized doubleblind placebo-controlled study. Mayo Clin Proc. 2008;83(9):1002-10.

17. Elhilali M, Ramsey E, Barkin J, Casey R, Boake $\mathrm{R}$, Beland $\mathrm{G}$, et al. A multicenter, randomized, double-blind, placebo-controlled study to evaluate the safety and efficacy of terazosin in the treatment of benign prostatic hyperplasia. Urology. 1996;47:335-42.

18. Roehrborn C. Efficacy and safety of once-daily alfuzosin in the treatment of lower urinary tract symptoms and clinical benign prostatic hyperplasia: a randomized, placebo-controlled trial. Urology. 2001;58:953-9.

19. Roehrborn C, Siegel R. Safety and efficacy of doxazosin in benign prostatic hyperplasia: a pooled analysis of three double-blind, placebocontrolled studies. Urology. 1996;48:406-15.

20. Debruyne F. Alpha blockers: are all created equal?. Urology. 2000; 56(5 suppl 1):20-2.

21. Roehrborn C, Van Kerrebroeck P, Nordling J. Safety and efficacy of alfuzosin $10 \mathrm{mg}$ once-daily in the treatment of lower urinary tract symptoms and clinical benign prostatic hyperplasia: a pooled analysis of three double-blind, placebo-controlled studies. BJU Int. 2003;92:257-61.

22. Lepor H. Phase III multicenter placebo-controlled study of tamsulosin in benign prostatic hyperplasia. Urology. 1998;51:892-900.

23. McConnell J. Medical management of benign prostatic hyperplasia with androgen suppression. Prostate. 1990;3(Suppl):49-59.

24. Kaplan S, McConnell J. Combination therapy with doxazosin and finasteride for benign prostatic hyperplasia in patients with lower urinary tract symptoms and a baseline total prostate volume of $25 \mathrm{~mL}$ or greater. J Urol. 2006;175(1):217-20.

25. Boyle P, Gould A, Roehrborn C. Prostate volume predicts outcome of treatment of benign prostatic hyperplasia with finasteride: meta-analysis of randomized clinical trials. Urology. 1996;48:398405.

26. The Finasteride Study Group. Finasteride (MK906) in the treatment of benign prostatic hyperplasia. Prostate. 1993;22:291-9.

27. Gormley G, Stoner E, Bruskewitz R, ImperatoMcGinley J, Walsh P, McConnell JD, et al. The effect of finasteride in men with benign prostatic hyperplasia. N Engl J Med. 1992;327:1185-91.

28. Marberger MJ, PROWESS Study Group. Longterm effects of finasteride in patients with benign prostatic hyper-plasia: a double-blind, placebo-controlled, multi-center study. Urol, 1998; 51:677-86. 
*29. McConnell J, Bruskewitz R, Walsh P, Andriole $\mathrm{G}$, Lieber M, Holtgrewe $\mathrm{H}$, et al. The effect of finasteride on the risk of acute urinary retention and the need for surgical treatment among men with benign prostatic hyperplasia. N Engl J Med. 1998;338:557-63.

30. Roehrborn C, Boyle P, Nickel J, Hoefner K, Andriole G, et al. ARIA3002, and ARIA3003 Study In-vestigators. Efficacy and safety of a dual inhibitor of 5-alpha-reductase types 1 and 2 (dutasteride) in men with benign prostatic hyperplasia. Urology. 2002;60:434-41.

31. Hyman M, Groutz A, Blaivas J. Detrusor instability in men: correlation of lower urinary tract symptoms with urodynamic findings. J Urol. 2001;166:550-2.

32. Laniado M, Ockrim J, Marronaro A, Tubaro A, Carter S. Serum prostate specific antigen to predict the presence of bladder outlet obstruction in men with urinary symptoms. BJU International. 2004;94:1283-6.

33. Van Kerrebroeck P, Kreder K, Jonas U, Zinner N, Wein A. Tolterodine once-daily: superior efficacy and tolerability in the treatment of overactive bladder. Urology. 2001;57:414-21.

34. Cardozo L, Lisec M, Millard R, van Vierssen Trip, et al. Randomized double-blind placebo controlled trial of the once daily antimuscarinic agent solifenacin succinate in patients with overactive bladder. J Urol. 2004;1:1919-1924.

35. Kirby R, Roehrborn C, Boyle P, Bartsch G, Jardin A, Cary MM, et al. for the Prospective European Doxazosin and Combination Therapy Study Investigators. Efficacy and tolerability of doxazosin and finasteride, alone or in combination, in treatment of symptomatic benign prostatic hyperplasia: the Prospective European Doxazosin and Combination Therapy (PREDICT) trial. Urology. 2003;61:119-26.

36. Kaplan S, McConnell J, Roehrborn C, Meehan A, Lee M, Noble W, et al. Combination Therapy With Doxazosin and Finasteride for Benign Prostatic Hyperplasia in Patients With Lower Urinary Tract Symptoms and a Baseline Total Prostate Volume of $25 \mathrm{~mL}$ or Greater J Urol. 2006;175(1):217-20.

37. Barkin J, Guimaraes M, Jacobi G, Pushkar D, Taylor S, van Vierssen Trip O, et al. Alpha-blocker therapy can be withdrawn in the majority of men following initial combination therapy with the dual 5alpha-reductase inhibitor dutasteride. Eur Urol. 2003;44:461-6.

38. Roehrborn C, Siami P, Barkin J, Damiao R, Major-Walker K, Morrill B, et al. The effects of dutasteride, tamsulosin and combination therapy on lower urinary tract symptoms in men with benign prostatic hyperplasia and prostatic enlargement: 2-year results from the CombAT study. J Urol. 2008;179:616-21.

39. Athanasopoulos A, Gyftopoulos K, Giannitsas K, Fisfis J, Perimenis P, Barbalias G. Combination treatment with an alpha-blocker plus an anticholinergic for bladder outlet obstruction: a prospective, randomized controlled trial. J Urol. 2003;169:2253-6.

40. Lee K, Choo M, Kim D, Kim J, Min K, Lee J, et al. Combination treatment with propiverine hydrochloride plus doxazosin controlled release gastrointestinal therapeutic system formulation for overactive bladder and coexisting benign prostatic obstruction: a prospective, randomized, controlled multicenter study. J Urol. 2005;174:1334-8.

41. Kaplan S, Roehrborn C, Rovner E, Carlsson M, Bavendam T, Guan Z. Tolterodine and tamsulosin for treatment of men with lower urinary tract symptoms and overactive bladder: a randomized controlled trial. JAMA. 2007;296(19):2319-28.

42. Kaplan S, Roehrborn C, Chancellor M, Carlsson M, Bavendam T, Guan Z. Extended-release tolterodine with or without tamsulosin in men with lower urinary tract symptoms and overactive bladder: effects on urinary symptoms assessed by the international prostate symptom score. BJU International. 2008;102(9):1133-9.

43. Chapple C, Herschorn S, Abrams P, Sun F, Brodsky M, Guan Z. Tolterodine treatment improves storage symptoms suggestive of overactive bladder in men treated with alpha-blockers. Eur Urol, 2009; 56(3):534-41

44. Rovner E, Kreder K, Sussman D, Kaplan S, Carlsson M, Bavendam T, et al. Effect of tolterodine extended release with or without tamsulosin on measures of urgency and patient reported outcomes in men with lower urinary tract symptoms. J Urol. 2008;180(3):1034-41.

45. Roehrborn C, Kaplan S, Kraus S, Wang J, Bavendam T, Guan Z. Effects of serum PSA on efficacy of tolterodine extended release with or without tamsulosin in men with LUTS including OAB. Urology. 2008;72(5):1061-7.

46. Roehrborn C, Kaplan S, Jones J, Wang J, Bavendam T, Guan Z. Tolterodine extended release with or without tamsulosin in men with lower urinary tract symptoms including overactive bladder symptoms: effect of prostate size. Eur Urol, 2009; 55(2):472-479.

47. Chung D, Te A, Staskin D, Kaplan S. Efficacy and safety of tolterodine extended release and dutasteride in male overactive bladder patients with prostates $\geq 30$ grams. Urol, 2010; 75:1144-8. 